\title{
Usefulness of matrix-assisted laser desorption ionization time-of-flight mass spectrometry to identify pathogens, including polymicrobial samples, directly from blood culture broths
}

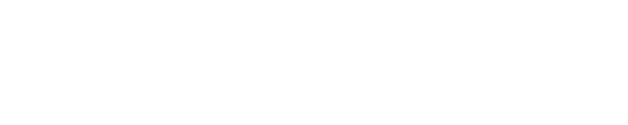

\author{
Maya Hariu ${ }^{1,2}$ \\ Yuji Watanabe ${ }^{1,2}$ \\ Nozomi Oikawa ${ }^{1,2}$ \\ Masafumi Seki' \\ 'Division of Infectious Diseases and \\ Infection Control, ${ }^{2}$ Laboratory for \\ Clinical Microbiology, Tohoku Medical \\ and Pharmaceutical University \\ Hospital, Sendai, Japan
}

\begin{abstract}
Matrix-assisted laser desorption ionization time-of-flight mass spectrometry (TOFMS) is now widely used to detect pathogens in clinical settings in Japan. Here, we report the ability of TOF-MS to detect bacteria from blood culture (BC) broths, and compare the efficacy of TOF-MS to that of conventional culture methods. Bacteria were correctly detected from 63 monomicrobial samples within 80 minutes; results matched those obtained by conventional $\mathrm{BC}$ methods, although the conventional methods took 2-3 days. In addition to the 63 monomicrobial samples, another three polymicrobial samples were tested; notably, the infecting bacteria were not correctly identified in two of these three samples. To better assess the TOF-MS detection of polymicrobial samples, we tested various ratios of mixed broth samples, including combinations of the bacteria that we were unable to detect in clinical samples. Combinations of Enterobacter cloacae and Pseudomonas aeruginosa were correctly detected at a culture ratio of 2:1, but not in the 3:1 mixture. These results suggested that TOF-MS is a strong tool for the rapid and correct detection of pathogens from monomicrobial BC samples, though results need to be carefully checked when handling known or suspected polymicrobial samples.
\end{abstract}

Keywords: bacteremia, polymicrobial sample, Enterobacter cloaca, Pseudomonasa aeruginosa

\section{Introduction}

Sepsis, a host-wide infection by bacteria, often presents as a multiple-organ dysfunction and results in a 10-40\% mortality rate. Bacteremia is typically diagnosed by microbiological tests, usually consisting of blood cultures (BCs). ${ }^{1}$ The presence of pathogens in the bloodstream is detected in only $4-12 \%$ of all BCs in hospitals; the identification of pathogens via BC can take 2-3 days and sometimes yields false-negative results. ${ }^{1,2}$ Therefore, rapid bacterial identification from $\mathrm{BCs}$ is of great interest, especially for sepsis cases. Examples of other identification techniques include polymerase chain reaction, fluorescence in situ hybridization, and high-throughput DNA sequencing. ${ }^{2-5}$

Matrix-assisted laser desorption ionization time-of-flight mass spectrometry (TOF-MS) allows identification of most of the pathogenic bacteria grown on agar plates from isolated colonies within a few minutes, and has proven efficiency and reproducibility. ${ }^{6-8}$ Plasma represents up to $55 \%$ of the total blood volume, and offers a good opportunity to recover grown bacteria, as microbes are mostly present in the extracellular compartment.

In this study, we performed identification of bacteria in BCs by TOF-MS, and compared our results with those obtained by conventional BC methods. Among the
Correspondence: Masafumi Seki Division of Infectious Diseases and Infection Control, Tohoku Medical and Pharmaceutical University Hospital, I-I2-I Fukumuro, Miyagino-ku, Sendai, Miyagi 983-86/2, Japan

Tel $+8|22983| 22 \mid$

Fax $+8 \mid 22983$ I232

Email seki@hosp.tohoku-mpu.ac.jp 
clinical samples tested, few contained two distinct bacterial species, but the polymicrobial samples represented the biggest challenge for TOF-MS identification. Therefore, we further examined several combinations of bacteria at various ratios in an effort to better assess the utility of TOF-MS for such samples.

\section{Patients and methods Samples}

Blood specimens from adult patients were systematically collected and cultured using the Signal Blood Culture System (Oxoid; Thermo Fisher Scientific, Waltham, MA, USA), including both anaerobic and aerobic resins. Bottles containing 8-10 mL of whole blood were incubated in MIR553 instruments (SANYO, Moriguchi, Japan) for 5-7 days for the standard incubation protocol, or for fewer days if an earlier positive signal was detected. The direct identification of bacteria by TOF-MS from both positive aerobic and anaerobic bottles (Bruker Daltonics, Billerica, MA, USA) was performed serially in parallel with the routine protocol at the clinical laboratory of Tohoku Medical and Pharmaceutical University Hospital; these tests were performed from July 2014 to June 2015. After subculturing on agar plates, any positive $\mathrm{BC}$ was simultaneously assessed for bacterial identification using conventional procedures, including Walkaway panels (Beckman Coulter, Brea, CA, USA), and if necessary, with complementary individual biochemical or enzymatic tests.

This study was approved by the Committee for Clinical Scientific Research of Tohoku Medical and Pharmaceutical University Hospital in July 08 and December 10, 2015 (No. ID2015-2-008 and ID2015-2-037, respectively), and all patients whose blood specimens were used provided written informed consent.

\section{Separation of blood cells and bacteria and protein extraction protocol and identification of bacteria}

We used the MALDI Sepsityper kit (Bruker Daltonics). In brief, approximately $1 \mathrm{~mL}$ of cultured sample was recovered from a given positive $\mathrm{BC}$ bottle, transferred to a microfuge tube, and combined with $200 \mu \mathrm{L}$ lysis buffer. After mixing by vortexing for 10 seconds, tubes were centrifuged at $10,000 \mathrm{~g}$ for 2 minutes at room temperature, and supernatants were removed. We then resuspended each pellet by pipetting with $1 \mathrm{~mL}$ washing buffer before recentrifuging the samples for 1 minute at $10,000 \mathrm{~g}$. After removal of the supernatant, the bacterial pellet was treated according to the standard ethanol/formic acid protein extraction protocol for TOF-MS identification according to MBT Sepsityper kit instruction manual.

\section{Mixture of bacteria in various ratios for analysis by TOF-MS}

To examine the ability of TOF-MS to distinguish the polymicrobial samples, we prepared several types of bacterial mixtures in various ratios, ranging from 1:9 to $4: 1$, and then performed the $\mathrm{BC}$ broth analysis as described. The selected bacteria were the following: a: Escherichia coli, b: Pseudomonas aeruginosa, c: Enterococcus faecalis, d: Staphylococcus aureus, e: Streptococcus pneumoniae, f: Staphylococcus epidermidis, and g: Enterobacter cloacae. The combinations were as follows: $1: \mathrm{a}+\mathrm{b}, 2: \mathrm{a}+\mathrm{c}, 3: \mathrm{a}+\mathrm{d}, 4: \mathrm{a}+\mathrm{e}, 5: \mathrm{a}+\mathrm{f}, 6: \mathrm{b}+\mathrm{c}$, 7: b+d, 8: b+e, 9: b+f, 10: b+g, 11: c+d, 12: c+e, 13: c+f, 14: $d+e, 15: d+f, 16: e+f$, and $17: f+g$.

\section{Results \\ Detection of pathogens from the patient samples by TOF-MS}

We tested 463 blood samples originating from anaerobic/ aerobic blood vials. For this comparison of TOF-MS results with those obtained by routine techniques, 66 samples were positive for bacteria, and 20 distinct species were detected in the overall study (Tables 1 and 2). MALDI-TOF BioTyper was able to identify most or all of these specimens at the species level.

A total of 63 samples were monomicrobial cases, with $E$. coli $(\mathrm{n}=18)$, S. aureus $(\mathrm{n}=10)$, and $S$. epidermidis $(\mathrm{n}=8)$ detected most frequently (Table 1). Average score values were 2.465 for E. coli, and $>2.0$ for both $S$. aureus and S. epidermidis. For all the bacteria-positive sample, average scores were 2.408 for Gram-negative rods and 2.281 for Gram-positive cocci.

\section{Polymicrobial BCs}

Routine classical identification detected three polymicrobial samples representing six isolates; however, TOF-MS identified only four bacteria in these three samples using the identification methods provided by BioTyper (Table 2). Specifically, BioTyper called two of these three cases as monomicrobial samples (Case 2 and Case 3; Table 2).

In fact, polymicrobial samples were suspected when MALDI BioTyper proposed two alternative species that were identified with score values $>1.7$. For such cases, BioTyper allows the superimposition and comparison of the sample spectrum with library spectra in various mixture ratios (Figure 1).

We detected both E. cloacae and P. aeruginosa (Case 2, Table 2) from clinical samples when we mixed at 2:1 
Table I Bacteria detected from blood culture broth by time-offlight mass spectrometry matched with the conventional method

\begin{tabular}{lll}
\hline Detected pathogens $\mathbf{( n = 6 3 )}$ & Number & Score value \\
\hline Escherichia coli & 18 & 2.465 \\
Staphylococcus aureus & 10 & 2.387 \\
Staphylococcus epidermidis & 8 & 2.141 \\
Enterococcus faecalis & 3 & 2.426 \\
Klebsiella pneumoniae & 3 & 2.226 \\
Pseudomonas aeruginosa & 3 & 2.399 \\
Staphylococcus caprae & 2 & 2.175 \\
Staphylococcus hominis & 2 & 2.201 \\
Streptococcus agalactiae Group B & 2 & 2.461 \\
Streptococcus constellatus & 2 & 2.199 \\
Aerococcus urinae & $\mathrm{I}$ & 2.149 \\
Corynebacterium striatum & $\mathrm{I}$ & 2.304 \\
Enterobacter agglomerans & $\mathrm{I}$ & 2.374 \\
Enterobacter cloacae & $\mathrm{I}$ & 2.283 \\
Klebsiella ornithinolytica & $\mathrm{I}$ & 2.380 \\
Proteus mirabilis & $\mathrm{I}$ & 2.395 \\
Staphylococcus capitis & $\mathrm{I}$ & 2.179 \\
Streptococcus anginosus & $\mathrm{I}$ & $2.45 \mathrm{I}$ \\
Streptococcus bovis & $\mathrm{I}$ & 2.355 \\
Streptococcus pyogenes (Group A) & $\mathrm{I}$ & 2.289 \\
\hline
\end{tabular}

Table 2 Detected or non-detected polymicrobial cases by timeof-flight mass spectrometry (TOF-MS)

\begin{tabular}{llll}
\hline Case & $\begin{array}{l}\text { Pathogens detected by } \\
\text { conventional culture }\end{array}$ & $\begin{array}{l}\text { Pathogens detected } \\
\text { by TOF-MS }\end{array}$ & $\begin{array}{l}\text { Score } \\
\text { value }\end{array}$ \\
\hline I & $\begin{array}{l}\text { Streptococcus constellatus }+ \\
\text { Klebsiella pneumoniae }\end{array}$ & $\begin{array}{l}\text { S. constellatus }+ \\
\text { K. pneumoniae }\end{array}$ & 2.104 \\
2 & $\begin{array}{l}\text { Enterobacter cloacae }+ \\
\text { E. cloacae }\end{array}$ & 2.325 \\
3 & $\begin{array}{l}\text { Pseudomonas aeruginosa } \\
\text { Staphylococcus epidermidis } \\
\text { Enterococcus faecalis }\end{array}$ & S. epidermidis & 2.167 \\
\hline
\end{tabular}

ratio (Figure 1A), but E. cloacae alone in the 3:1 mixture (Figure 1B). Therefore, we next performed TOF-MS analysis on samples that combined several pairs of bacteria at various mixture ratios, including the same combinations as detected in Cases 2 and 3 (Table 3). We found that TOF-MS was able to detect the combined bacteria at ratios ranging from 1:3 to $1: 1$ (Table 3 ).

\section{Discussion}

New technologies, such as TOF-MS, have revolutionized the identification of bacteria. TOF-MS is fast, reliable, and inexpensive, and the majority of clinically relevant bacteria can be identified at the species level. ${ }^{6-8}$

In contrast, even though $\mathrm{BC}$ is considered to be the standard for the detection of microorganisms in blood samples, identification by $\mathrm{BC}$ remains dependent on several different factors, including bacterial density, bacterial adaptation to new growth environments, individual rates of growth, competition between bacteria, and initial antimicrobial therapy. ${ }^{2,9}$ Any delay in identification remains unsatisfactory for clinicians, who may be forced to initiate antimicrobial therapy prior to precise diagnosis. ${ }^{1}$ Shortening the delay in providing appropriate therapy may strengthen therapeutic specificity, and is expected to have a beneficial effect on the recovery of patients. The high sensitivity of TOF-MS in detecting peptides and the possibility of identifying bacteria with software such as BioTyper has been shown to permit high-quality microbial identification by TOF-MS. ${ }^{10}$

Here, we demonstrated the identification of bacteria from BCs using MALDI-TOF BioTyper, which allowed 95.5\% correct, single-step identifications among a total of 20 microorganisms from 66 clinical blood samples, including three polymicrobial samples, starting from small volumes of BC. Monomicrobial samples were correctly identified at the species level in $100 \%$ of cases. All bacteria were identified within the first 2-3 hours following BC positivity.

Hansen et al reported that TOF-MS analysis did not produce scores high enough for species identification in two bacteremia cases that presented with diverticular diseases; instead, the infecting bacteria were identified by the sequencing method, ${ }^{11}$ as we have previously reported. ${ }^{3,12,13}$ However, the infecting bacteria investigated by Hansen et al were relatively rare Gram-positive cocci, such as Ruminococcus species. ${ }^{11}$ Furthermore, treatment following rapid organism identification by TOF-MS has been shown to lead to reduced mortality in severe sepsis cases after implementation of TOF-MS in combination with BC. ${ }^{14}$ Furthermore, TOF-MS recently has been shown to reduce the use of wide-spectrum antibiotics in infection control when employed as part of an antimicrobial stewardship program. ${ }^{15}$ These results suggest that rapid and accurate identification of microbes by TOF-MS can contribute to better treatment and outcomes for patients with infectious diseases in clinical settings.

However, identification by TOF-MS is limited by the species and some specific situations; notably, polymicrobial cases may not be correctly diagnosed by TOF-MS. For polymicrobial samples, the observed profile may represent the mixed profiles of two distinct bacteria, with both showing significant scores (Table 2). Such a situation will require closer examination in the TOF-MS context. In these cases, the corresponding BCs will need to be carefully checked at the next isolation plate (typically grown for testing antimicrobial susceptibility), to distinguish the presence of additional bacterial isolates for subsequent identification, if necessary. This follow-up evaluation may help to validate the initial status of the blood samples, if not precluded earlier by Gram staining. 
A $\quad+$ Pseudomonas aeruginosa 19955

Enterobacter cloacae 20105
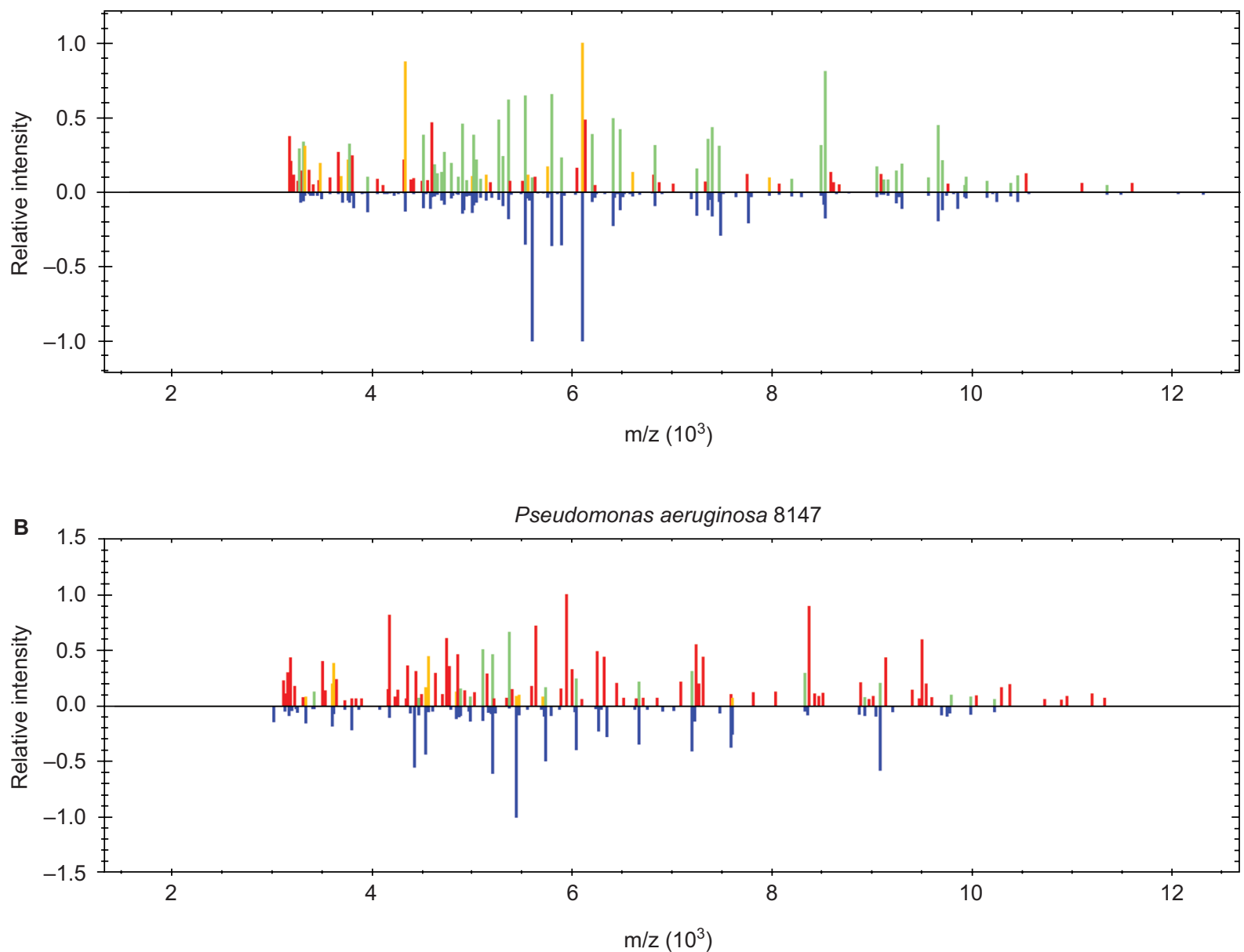

Figure I Identification of polymicrobial results displayed within the BioTyper 2.0 graphic view in the case of Enterobacter cloacae and Pseudomonas aeruginosa at ratios of 2:I (A) and 3:I (B), respectively. The mass spectra show the difference in peaks (presence or absence) and their intensities between the sample spectrum and those of bacteria identified at first pass by BioTyper. The upper part of the figure within the inner windows shows the unknown spectrum containing perfectly matching peaks (0-200 ppm) in green, imperfectly matching peaks (200-500 ppm) in yellow, and non-matching peaks in red. The lower part (blue) shows the dedicated main spectrum included in the database. In our case, both $E$. cloacae and $P$. aeruginosa were identified correctly at a score value of 2.580 in the $2: 1$ mixture ratio (A), but only $E$. cloacae was identified (at a score value of 2.407$)$ in the $3: 1$ mixture ratio $(\mathbf{B})$.

Christner et al reported that BioTyper scores $>1.5$ were essential for the identification of $8 \%$ of the isolates, but that work did not consider the possibility of polymicrobial samples. ${ }^{16}$ Moussaoui et al tested a new protocol for bacterial identification from BC broths, but only 10 of a total of 50 isolates from 21 polymicrobial samples were identified in that work. ${ }^{6}$ La Scola and Raoult reported the identification of only one of each mixture of species for 18 samples among 22 bacteria-positive $\mathrm{BC}$ broths that contained two or more different species. ${ }^{7}$ No species was identified in two of those polymicrobial samples, and false species identifications were obtained in two cases. ${ }^{7}$ Using an in-house saponin lysis method (in place of the MALDI Sepsityper kit), Meex et al were able to identify only one of each pair of isolates in six separate polymicrobial BCs. ${ }^{8}$ These results suggest that the identification by TOF-MS of two or more bacteria in polymicrobial samples is a challenge. Therefore, if the presence of more than one pathogen is suspected, it may be better to try to test the various mixture ratios, as demonstrated in the present study.

In conclusion, we examined the analytical ability of TOFMS and found that the technique yielded valid identification for $>95 \%$ of bacteria derived from monomicrobial and polymicrobial BC samples. Even in polymicrobial cases, analysis of corrected mixture ratios of combinations of the candidate infecting bacteria may facilitate detection of individual species, as we have shown in the present study. This rapid identification by TOF-MS is expected to lower costs for clinical laboratories and to provide better information to physicians, facilitating targeted antimicrobial therapy and potentially contributing to a decrease in antimicrobial pressure in hospitals. 
Table 3 Detected bacteria in various combination ratios by time-of-flight mass spectrometry

\begin{tabular}{|c|c|c|c|c|c|c|c|c|c|c|c|c|}
\hline \multirow[t]{2}{*}{ Combinations } & \multicolumn{12}{|c|}{ Ratio } \\
\hline & I:9 & $1: 8$ & $1: 7$ & I:6 & I:5 & I:4 & $\mathrm{I}: 3$ & $\mathrm{I}: 2$ & I:I & 2:1 & $3: 1$ & 4:1 \\
\hline I & $\mathrm{b}$ & $\mathrm{b}$ & $\mathrm{b}$ & $\mathrm{b}$ & $\mathrm{b}$ & Both & Both & Both & Both & Both & Both & a \\
\hline 2 & Both & Both & Both & Both & Both & Both & Both & Both & $\mathrm{a}$ & $\mathrm{a}$ & $\mathrm{a}$ & a \\
\hline 3 & d & d & d & d & d & Both & Both & Both & a & $\mathrm{a}$ & a & a \\
\hline 4 & e & Both & Both & Both & Both & Both & Both & Both & $\mathrm{a}$ & $\mathrm{a}$ & $\mathrm{a}$ & $\mathrm{a}$ \\
\hline 5 & $f$ & f & f & f & f & f & Both & Both & Both & $\mathrm{a}$ & a & a \\
\hline 6 & Both & Both & Both & Both & Both & Both & Both & Both & Both & Both & Both & $\mathrm{b}$ \\
\hline 7 & d & Both & Both & Both & Both & Both & Both & Both & Both & Both & Both & b \\
\hline 8 & e & e & e & e & Both & Both & Both & Both & Both & b & $\mathrm{b}$ & b \\
\hline 9 & $f$ & $f$ & $f$ & Both & Both & Both & Both & Both & Both & Both & Both & b \\
\hline 10 & $g$ & $g$ & $g$ & $g$ & $\mathrm{~g}$ & $g$ & $g$ & Both & Both & Both & Both & b \\
\hline II & d & d & d & d & d & d & Both & Both & Both & Both & Both & c \\
\hline 12 & e & e & e & e & e & Both & Both & Both & c & c & c & c \\
\hline 12 & f & $f$ & $f$ & $f$ & $f$ & f & Both & Both & Both & Both & Both & c \\
\hline 14 & e & e & e & e & e & Both & Both & Both & d & d & d & $d$ \\
\hline 15 & $f$ & Both & Both & Both & Both & Both & Both & Both & Both & Both & d & $d$ \\
\hline 16 & $f$ & f & f & $f$ & f & f & Both & Both & Both & Both & e & e \\
\hline 17 & $g$ & $g$ & $g$ & $g$ & $g$ & $g$ & $\mathrm{~g}$ & $g$ & $\mathrm{~g}$ & Both & Both & $f$ \\
\hline
\end{tabular}

Notes: a: Escherichia coli, b: Pseudomonas aeruginosa, c: Enterococcus faecalis, d: Staphylococcus aureus, e: Streptococcus pneumoniae, f: Staphylococcus epidermidis, and g: Enterobacter cloacae; I=a:b, 2=a:c, 3=a:d, 4=a:e, 5=a:f, 6=b:c, 7=b:d, 8=b:e, 9=b:f, I0=b:g, II=c:d, I2=c:e, I3=c:f, I4=d:e, I5=d:f, I6=e:f, and I7=f:g.

\section{Disclosure}

This work was supported by the Japanese Society for the Promotion of Science Grant-in-Aid for Scientific Research 26461158 (to MS). The authors report no conflicts of interest in this work.

\section{References}

1. Singer M, Deutschman CS, Seymour CW, et al. The third international consensus definitions for sepsis and septic shock (sepsis-3). JAMA. 2016;315:801-810.

2. Yanagihara K, Kitagawa Y, Tomonaga M, et al. Evaluation of pathogen detection from clinical samples by real-time polymerase chain reaction using a sepsis pathogen DNA detection kit. Crit Care. 2010;14(4):R159.

3. Seki M, Gotoh K, Nakamura S, et al. Fatal sepsis caused by an unusual Klebsiella species that was misidentified by an automated identification system. J Med Microbiol. 2013;62(Pt 5):801-803.

4. Seki M, Takahashi H, Yamamoto N, et al. Polymerase chain reactionbased active surveillance of MRSA in emergency department patients. Infect Drug Resist. 2015;8:113-118.

5. Ikeuchi T, Seki M, Akeda Y, et al. PCR-based method for rapid and minimized electrochemical detection of mecA gene of methicillinresistant Staphylococcus aureus and methicillin-resistant Staphylococcus epidermis. Glob J Infect Dis Clin Res. 2016;2(1):8-12.

6. Moussaoui W, Jaulhac B, Hoffmann AM, et al. Matrix-assisted laser desorption ionization time-of-flight mass spectrometry identifies $90 \%$ of bacteria directly from blood culture vials. Clin Microbiol Infect. 2010; 16(11):1631-1638.

7. La Scola B, Raoult D. Direct identification of bacteria in positive blood culture bottles by matrix-assisted laser desorption ionisation time-offlight mass spectrometry. PLoS One. 2009;4(11):e8041.
8. Meex C, Neuville F, Descy J, et al. Direct identification of bacteria from BacT/ALERT anaerobic positive blood cultures by MALDI-TOF-MS: MALDI Sepsityper kit versus an in-house saponin method for bacterial extraction. J Med Microbiol. 2012;61(Pt 11):1511-1516.

9. Schelonka RL, Chai MK, Yoder BA, Hensley D, Brockett RM, Ascher DP. Volume of blood required to detect common neonatal pathogens. J Pediatr. 1996;129(2):275-278.

10. Mellmann A, Bimet F, Bizet C, et al. High interlaboratory reproducibility of matrix-assisted laser desorption ionization-time of flight mass spectrometry-based species identification of nonfermenting bacteria. J Clin Microbiol. 2009;47(11):3732-3734.

11. Hansen SG, Skov MN, Justesen US. Two cases of Ruminococcus gnavus bacteremia associated with diverticulitis. J Clin Microbiol. 2013; 51(4):1334-1336.

12. Seki M, Ohno H, Gotoh K, et al. Allergic bronchopulmonary mycosis due to co-infection with Aspergillus fumigatus and Schizophyllum commune. IDCases. 2014;1(1):5-8.

13. Yoshida H, Seki M, Umeyama T, et al. Invasive pulmonary aspergillosis due to Aspergillus lentulus: successful treatment of a liver transplant patient. J Infect Chemother. 2015;21(6):479-481.

14. Carreno JJ, Lomaestro BM, Jacobs AL, Meyer RE, Evans A, Montero CI. Assessment of time to clinical response in patients with sepsis treated before and after implementation of a matrix-assisted laser desorption ionization time-of-flight blood culture identification algorithm. Infect Control Hosp Epidemiol. 2016;37(8):916-923.

15. Buss BA, Schulz LT, Reed KD, Fox BC. MALDI-TOF utility in a region with low antibacterial resistance rates. Clin Infect Dis. 2016;62(5): 666-667.

16. Christner M, Rohde H, Wolters M, Sobottka I, Wegscheider K, Aepfelbacher M. Rapid identification of bacteria from positive blood culture bottles by use of matrix-assisted laser desorption-ionization time of flight mass spectrometry fingerprinting. J Clin Microbiol. 2010;48(5): 1584-1591. 


\section{Publish your work in this journal}

Infection and Drug Resistance is an international, peer-reviewed openaccess journal that focuses on the optimal treatment of infection (bacterial, fungal and viral) and the development and institution of preventive strategies to minimize the development and spread of resistance. The journal is specifically concerned with the epidemiology of antibiotic resistance and the mechanisms of resistance development and diffusion in both hospitals and the community. The manuscript management system is completely online and includes a very quick and fair peerreview system, which is all easy to use. Visit http://www.dovepress.com/ testimonials.php to read real quotes from published authors.

Submit your manuscript here: https://www.dovepress.com/infection-and-drug-resistance-journal 